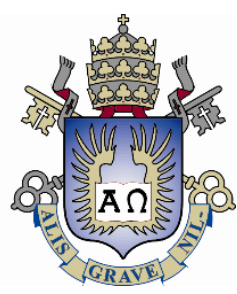

Leonardo Chagas Carbone

Simulação numérica de um misturador gáslíquido para estações de bombeio

Dissertação de Mestrado

Dissertação apresentada como requisito parcial para obtenção do título de Mestre pelo Programa de PósGraduação em Engenharia Mecânica da PUC-Rio.

Orientadora: Professora Angela Ourivio Nieckele 


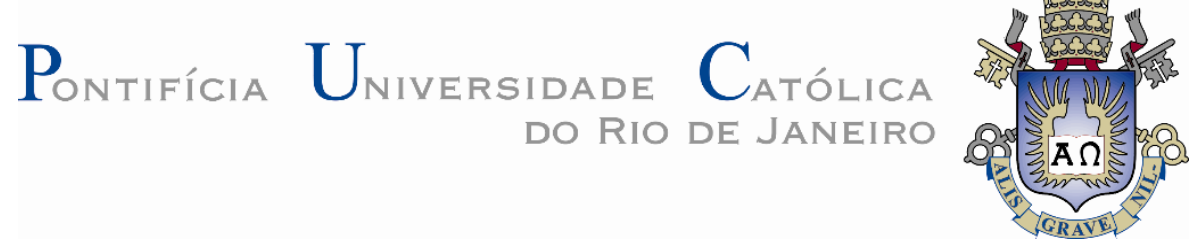

Leonardo Chagas Carbone

\section{Simulação numérica de um misturador gás- líquido para estações de bombeio}

Dissertação apresentada como requisito parcial para obtenção do título de Mestre pelo Programa de PósGraduação em Engenharia Mecânica da PUC-Rio. Aprovada pela Comissão Examinadora abaixo assinada.

Profa. Angela Ourivio Nieckele Orientadora Pontifícia Universidade Católica do Rio de Janeiro

Prof. Rigoberto Eleazar Melgarejo Morales Universidade Tecnológica Federal do Paraná

Prof. Igor Braga

Pontifícia Universidade Católica do Rio de Janeiro

Prof. José Eugenio Leal Coordenador Setorial do Centro

Técnico Científico - PUC-Rio 
Todos os direitos reservados. É proibida a reprodução total ou parcial do trabalho sem autorização da universidade, do autor e do orientador.

\section{Leonardo Chagas Carbone}

Graduou-se em Engenharia Mecânica na UFF no ano de 1997, especializou-se em Engenharia de Petróleo pela Universidade PETROBRAS em 2000 e atualmente trabalha na PETROBRAS na área de Elevação Artificial e Garantia de Escoamento

Ficha Catalográfica

Carbone, Leonardo Chagas

Simulação numérica de um misturador gás-líquido para estações de bombeio / Leonardo Chagas Carbone; orientadora: Angela Ourivio Nieckele. - Rio de Janeiro: PUC, Departamento de Engenharia Mecânica, 2015.

$136 \mathrm{f.} \mathrm{;} 30 \mathrm{~cm}$

Dissertação (mestrado) - Pontifícia Universidade Católica do Rio de Janeiro, Departamento de Engenharia Mecânica.

Inclui referências bibliográficas.

1. Engenharia mecânica - Teses. 2. Misturador. 3. Escoamento bifásico. 4. Escoamento intermitente. 5 . Sistema de bombeamento. 6. Simulação CFD. I. Nieckele, Angela Ourivio. II. Pontifícia Universidade Católica do Rio de Janeiro. Departamento de Engenharia Mecânica. III. Título. 
À minha esposa, que neste período me deu meus maiores presentes...

Arthur e Laís! 


\section{Agradecimentos}

À Profa. Angela Ourivio Nieckele, pela orientação, dedicação e paciência sem as quais este trabalho não poderia ter sido realizado.

Aos meus pais, que foram fundamentais na minha educação e na formação de meu caráter.

Aos colegas do CENPES Roberto da Fonseca Júnior e Luiz Carlos Tosta da Silva, por me apresentarem este tema e me incentivarem a estudá-lo.

À PETROBRAS pelo patrocínio e ao meu gerente Leonardo B. Testi, pela compreensão nos momentos em que precisei me dedicar a este trabalho.

Aos meus colegas de PETROBRAS pelo companheirismo e ajuda.

À minha família, Amanda, Arthur e Laís; por darem sentido à minha vida... 


\section{Resumo}

Carbone, Leonardo Chagas; Nieckele, Angela Ourivio. Simulação numérica de um misturador gás-líquido para estações de bombeio. Rio de Janeiro, 2015. 136p. Dissertação de Mestrado - Departamento de Engenharia Mecânica, Pontifícia Universidade Católica do Rio de Janeiro.

A produção de petróleo offshore tem requerido cada vez mais a utilização de equipamentos de bombeio no leito marinho para a produção de poços a longas distâncias das unidades de produção. A grande maioria destes equipamentos tem dificuldade de operar com a presença de gás, ainda mais em condições de golfadas, onde a fração de vazio na sucção pode variar significativamente. Isto limita enormemente a janela operacional dos equipamentos. Uma forma de minimizar os efeitos das golfadas e, assim, ampliar a janela de operação destes equipamentos é a instalação de misturadores de fluxo na sucção das bombas, que atuam de forma a garantir uma maior sobrevida ao equipamento. Portanto, o projeto e a previsão do comportamento deste tipo de equipamento são fundamentais para bom funcionamento dos sistemas de bombeamento submarino. O presente trabalho investiga numericamente, com o modelo de dois fluidos, a eficiência de um tipo de misturador em uniformizar as frações de vazio. Diferentes configurações geométricas são analisadas e a influência das vazões de admissão de ambas as fases é discutida. A metodologia é validada através da comparação da queda de pressão através do misturador com dados experimentais disponíveis, onde se observou boa concordância. O modelo numérico mostra ainda que uma reserva de líquido é mantida no interior do equipamento e, o gás é forçado a se dispersar na corrente de líquido para sair do misturador. $\mathrm{O}$ comportamento do misturador frente à presença de golfadas também é investigado. Ao final do trabalho é proposta uma nova geometria, que se mostra mais tolerante a intermitências no escoamento.

\section{Palavras-chave}

Misturador; escoamento bifásico; escoamento intermitente; sistema de bombeamento; simulação CFD. 


\section{Abstract}

Carbone, Leonardo Chagas; Nieckele, Angela Ourivio (Advisor). Numerical simulation of a gas-liquid mixer for pumping stations Rio de Janeiro, 2015. 136p. MSc. Dissertation - Departamento de Engenharia Mecânica, Pontifícia Universidade Católica do Rio de Janeiro.

The offshore oil production is increasingly using pumping equipment at the seabed for production of wells at long distances from production units. Most of these devices have difficulty to operate in the presence of gas, especially in slug flow conditions, where the pump suction's void fraction can vary significantly. This problem limits the equipment's range of operation. One way to minimize the effects of slugging and thus enlarge the operation range of these devices is to use flow mixers at the pump's suction, which helps to ensure the operation even in the presence of large gas bubbles. Therefore, the design and prediction of the behavior of this type of equipment are essential for proper operation of subsea pumping systems. At the present work, the efficiency of a type of flow mixer to provide uniform void fractions at its outlet is numerically investigated, with the two fluid model. Different geometric configurations are analyzed and the influence of intake flow of both phases is discussed. The methodology is validated through comparison of the pressure drop through the flow mixer with available experimental data, where good agreement was observed. The numerical model also shows that a buffer of liquid is maintained inside the equipment and the gas is forced to disperse in the liquid stream to exit the mixer. The mixer's behavior in the presence of slugs is also investigated. Finally, a new geometry is proposed, which seems to be more efficient to avoid flow's intermittencies.

\section{Keywords}

Flow mixer; two-phase flow; slug flow; pumping system; CFD simulation. 


\section{Sumário}

1 Introdução $\quad 21$

1.1 Benefícios do Uso de Métodos Bombeados 21

1.2 Desafios do Bombeamento Multifásico 24

$\begin{array}{ll}1.3 \text { Objetivo } & 27\end{array}$

$\begin{array}{ll}1.4 \text { O Misturador } & 27\end{array}$

$\begin{array}{ll}1.5 \text { Revisão Bibliográfica } & 30\end{array}$

1.6 Organização do Manuscrito 32

2 Modelagem Matemática 33

2.1 Forças Interfaciais de Transferência de Quantidade de Movimento Linear $\quad 35$

2.2 Distribuição da fase dispersa $\quad 41$

$\begin{array}{ll}2.3 \text { Turbulência } & 46\end{array}$

3 Modelagem Numérica $\quad 50$

3.1 Método de Volumes Finitos $\quad 51$

3.2 Definição da Geometria de Interesse 53

$\begin{array}{ll}3.3 \text { Estudo de Malha } & 56\end{array}$

4 Avaliação de Modelos Interfaciais 62

4.1 Resultados Experimentais 62

4.2 Caso 1, Bolhas Monodispersas, com Somente Força de Arraste 64

4.3 Caso 2, Bolhas Monodispersas, com Força de Sustentação 72

4.4 Caso 3, Bolhas Polidispersas, com Pequena Faixa de Diâmetros 78

4.5 Caso 4, Bolhas Polidispersas, com Grande Faixa de Diâmetros 86

5 Golfadas 105

5.1 Configurações 1 e $3 \quad 109$

5.2 Proposta de Nova Geometria 119

6 Comentários Finais 130

6.1 Sugestão de trabalhos futuros 132

Referências Bibliográficas 133 


\section{Lista de tabelas}

Tabela 1.1 - Configuração dos furos internos 30

Tabela 1.2 - Erro no hold-up de ar (Riera-Ortiz et al, 2011) 32

Tabela 2.1 - Constantes do modelo $\kappa-\omega$ SST 49

Tabela 3.1 - Parâmetros numéricos utilizados nas simulações $\quad 52$

Tabela 3.2 - Condições de contorno para os testes de malha 53

Tabela 3.3 - Número de nós e elementos 60

Tabela 3.4 - Configurações finais das malhas 61

Tabela 4.1 - Resultados experimentais 63

Tabela 4.2 - Distribuição dos diâmetros das bolhas no caso $4 \quad 98$

Tabela 5.1 - Condições de contorno para a simulação de golfadas 109 


\section{Lista de figuras}

Figura 1.1 - Recordes mundiais de distância entre unidades de produção e poços (OffShore Magazine pôster, 2014)

Figura 1.2 - Efeito de um tanque de amortecimento no torque de eixo (Hua et al., 2011)

Figura 1.3 - Vista lateral do misturador com dimensões

Figura 1.4 - Vista lateral em corte do misturador com dimensões

Figura 1.5 - Projeto do misturador

Figura 2.1 - Correlação entre números adimensionais e formas das partículas. (Clift, Grace e Weber, 1978)

Figura 2.2 - Grupos de velocidade e subdivisão de classes de tamanhos de bolha do espectro de tamanho de bolhas no modelo MUSIG (Frank, 2008)

Figura 3.1 - Resíduos RMS de quantidade de movimento e massa $\quad 52$

Figura 3.2 - Detalhes da malha gerada para a geometria completa $\quad 54$

Figura 3.3 - Fração de ar no modelo completo, vista em corte do misturador

Figura 3.4 - Gráficos de fração de ar ao longo do misturador 55

Figura 3.5 - Configuração da Malha 1

Figura 3.6 - Configuração da Malha $2 \quad 58$

Figura 3.7 - Configuração da Malha $3 \quad 58$

Figura 3.8 - Configuração da Malha $4 \quad 59$

Figura 3.9 - Configuração da Malha $5 \quad 59$

Figura 3.10 - Variação do diferencial de pressão com o número de elementos de cada malha

Figura 3.11 - Comparação da fração volumétrica de ar no plano de simetria

Figura 4.1 - Comparação entre as configurações analisadas do cilindro interno.

Figura 4.2 - Caso 1 - comparação entre os dados experimentais e numéricos. Somente força de arraste

Figura 4.3 - Caso 1, Configuração 1 - Fração volumétrica do ar no plano de simetria (a), entrada (b) e saída (c) do misturador para $U_{m}=$ $1,2 \mathrm{~m} / \mathrm{s}$

Figura 4.4 - Caso 1, Configuração 1 - Fração volumétrica do ar no plano de simetria (a), entrada (b) e saída (c) do misturador para $U_{m}=$ $1,4 \mathrm{~m} / \mathrm{s}$ 
Figura 4.5 - Caso 1, Configuração 1 - Fração volumétrica do ar no plano de simetria (a), entrada (b) e saída (c) do misturador para $U_{m}=$ $2,0 \mathrm{~m} / \mathrm{s}$

Figura 4.6 - Caso 1, Configuração 2 - Fração volumétrica do ar no plano de simetria (a), entrada (b) e saída (c) do misturador para $U_{m}=$ $1,2 \mathrm{~m} / \mathrm{s}$

Figura 4.7 - Caso 1, Configuração 2 - Fração volumétrica do ar no plano de simetria (a), entrada (b) e saída (c) do misturador para $U_{m}=$ $1,4 \mathrm{~m} / \mathrm{s}$

Figura 4.8 - Caso 1, Configuração 2 - Fração volumétrica do ar no plano de simetria (a), entrada (b) e saída (c) do misturador para $U_{m}=$ $2,0 \mathrm{~m} / \mathrm{s}$

Figura 4.9 - Caso 1, Configuração 3 - Fração volumétrica do ar no plano de simetria (a), entrada (b) e saída (c) do misturador para $U_{m}=$ $1,2 \mathrm{~m} / \mathrm{s}$

Figura 4.10 - Caso 1, Configuração 3 - Fração volumétrica do ar no plano de simetria (a), entrada (b) e saída (c) do misturador para $U_{m}=$ $1,4 \mathrm{~m} / \mathrm{s}$

Figura 4.11 - Caso 1, Configuração 3 - Fração volumétrica do ar no plano de simetria (a), entrada (b) e saída (c) do misturador para $U_{m}=$ $2,0 \mathrm{~m} / \mathrm{s}$

Figura 4.12 - Caso 1, Configuração 4 - Fração volumétrica do ar no plano de simetria (a), entrada (b) e saída (c) do misturador para $U_{m}=$ $1,2 \mathrm{~m} / \mathrm{s}$

Figura 4.13 - Caso 1, Configuração 4 - Fração volumétrica do ar no plano de simetria (a), entrada (b) e saída (c) do misturador para $U_{m}=$ $1,4 \mathrm{~m} / \mathrm{s}$

Figura 4.14 - Caso 1, Configuração 4 - Fração volumétrica do ar no plano de simetria (a), entrada (b) e saída (c) do misturador para $U_{m}=$ $2,0 \mathrm{~m} / \mathrm{s}$

Figura 4.15 - Influência do tamanho das bolhas

Figura 4.16 - Caso 2 - comparação entre os dados experimentais e numéricos. Presença de força de arraste e sustentação

Figura 4.17- Caso 2, Configuração 1 - Fração volumétrica do ar no plano de simetria (a), entrada (b) e saída (c) do misturador para $U_{m}=$ $1,2 \mathrm{~m} / \mathrm{s}$

Figura 4.18 - Caso 2, Configuração 1 - Fração volumétrica do ar no plano de simetria (a), entrada (b) e saída (c) do misturador para $U_{m}=$ $1,4 \mathrm{~m} / \mathrm{s}$

Figura 4.19 - Caso 2, Configuração 1 - Fração volumétrica do ar no plano de simetria (a), entrada (b) e saída (c) do misturador para $U_{m}=$ $2,0 \mathrm{~m} / \mathrm{s}$ 
Figura 4.20 - Caso 2, Configuração 2 - Fração volumétrica do ar no plano de simetria (a), entrada (b) e saída (c) do misturador para $U_{m}=$ $1,2 \mathrm{~m} / \mathrm{s}$

Figura 4.21 - Caso 2, Configuração 2 - Fração volumétrica do ar no plano de simetria (a), entrada (b) e saída (c) do misturador para $U_{m}=$ $1,4 \mathrm{~m} / \mathrm{s}$

Figura 4.22 - Caso 2, Configuração 2 - Fração volumétrica do ar no plano de simetria (a), entrada (b) e saída (c) do misturador para $U_{m}=$ $2,0 \mathrm{~m} / \mathrm{s}$

Figura 4.23 - Caso 2, Configuração 3 - Fração volumétrica do ar no plano de simetria (a), entrada (b) e saída (c) do misturador para $U_{m}=$ $1,2 \mathrm{~m} / \mathrm{s}$

Figura 4.24 - Caso 2, Configuração 3 - Fração volumétrica do ar no plano de simetria (a), entrada (b) e saída (c) do misturador para $U_{m}=$ $1,4 \mathrm{~m} / \mathrm{s}$

Figura 4.25 - Caso 2, Configuração 3 - Fração volumétrica do ar no plano de simetria (a), entrada (b) e saída (c) do misturador para $U_{m}=$ $2,0 \mathrm{~m} / \mathrm{s}$

Figura 4.26 - Caso 2, Configuração 4 - Fração volumétrica do ar no plano de simetria (a), entrada (b) e saída (c) do misturador para $U_{m}=$ $1,2 \mathrm{~m} / \mathrm{s}$

Figura 4.27- Caso 2, Configuração 4 - Fração volumétrica do ar no plano de simetria (a), entrada (b) e saída (c) do misturador para $U_{m}=$ $1,4 \mathrm{~m} / \mathrm{s}$

Figura 4.28 - Caso 2, Configuração 4 - Fração volumétrica do ar no plano de simetria (a), entrada (b) e saída (c) do misturador para $U_{m}=$ $2,0 \mathrm{~m} / \mathrm{s}$

Figura 4.29 - Caso 3 - Evolução temporal da fração de ar média no plano de saída do misturador, $U_{m}=1,4 \mathrm{~m} / \mathrm{s}$

Figura 4.30 - Caso 3 - comparação entre os dados experimentais e numéricos

Figura 4.31 - Comparação dos casos 1 a 3 com os dados experimentais

Figura 4.32 - Caso 3, Configuração 1 - Fração volumétrica do ar no plano de simetria (a), entrada (b) e saída (c) do misturador para $U_{m}=$ $1,2 \mathrm{~m} / \mathrm{s}$

Figura 4.33 - Caso 3, Configuração 1 - Fração volumétrica do ar no plano de simetria (a), entrada (b) e saída (c) do misturador para $U_{m}=$ $1,4 \mathrm{~m} / \mathrm{s}$

Figura 4.34 - Caso 3, Configuração 1 - Fração volumétrica do ar no plano de simetria (a), entrada (b) e saída (c) do misturador para $U_{m}=$ $2,0 \mathrm{~m} / \mathrm{s}$ 
Figura 4.35 - Caso 3, Configuração 2 - Fração volumétrica do ar no plano de simetria (a), entrada (b) e saída (c) do misturador para $U_{m}=$ $1,2 \mathrm{~m} / \mathrm{s}$

Figura 4.36 - Caso 3, Configuração 2 - Fração volumétrica do ar no plano de simetria (a), entrada (b) e saída (c) do misturador para $U_{m}=$ $1,4 \mathrm{~m} / \mathrm{s}$

Figura 4.37 - Caso 3, Configuração 2 - Fração volumétrica do ar no plano de simetria (a), entrada (b) e saída (c) do misturador para $U_{m}=$ $2,0 \mathrm{~m} / \mathrm{s}$

Figura 4.38 - Caso 3, Configuração 3 - Fração volumétrica do ar no plano de simetria (a), entrada (b) e saída (c) do misturador para $U_{m}=$ $1,2 \mathrm{~m} / \mathrm{s}$

Figura 4.39 - Caso 3, Configuração 3 - Fração volumétrica do ar no plano de simetria (a), entrada (b) e saída (c) do misturador para $U_{m}=$ $1,4 \mathrm{~m} / \mathrm{s}$

Figura 4.40 - Caso 3, Configuração 3 - Fração volumétrica do ar no plano de simetria (a), entrada (b) e saída (c) do misturador para $U_{m}=$ $2,0 \mathrm{~m} / \mathrm{s}$

Figura 4.41 - Caso 3, Configuração 4 - Fração volumétrica do ar no plano de simetria (a), entrada (b) e saída (c) do misturador para $U_{m}=$ $1,2 \mathrm{~m} / \mathrm{s}$

Figura 4.42 - Caso 3, Configuração 4 - Fração volumétrica do ar no plano de simetria (a), entrada (b) e saída (c) do misturador para $U_{m}=$ $1,4 \mathrm{~m} / \mathrm{s}$

Figura 4.43 - Caso 3, Configuração 4 - Fração volumétrica do ar no plano de simetria (a), entrada (b) e saída (c) do misturador para $U_{m}=$ $2,0 \mathrm{~m} / \mathrm{s}$

Figura 4.44 - Caso 4 - comparação entre os dados experimentais e numéricos

Figura 4.45 - Comparação do caso 4 com os dados experimentais

Figura 4.46 - Caso 4, Configuração 1 - Fração volumétrica do ar no plano de simetria (a), entrada (b) e saída (c) do misturador para $U_{m}=$ $1,2 \mathrm{~m} / \mathrm{s}$

Figura 4.47 - Caso 4, Configuração 1 - Fração volumétrica do ar no plano de simetria (a), entrada (b) e saída (c) do misturador para $U_{m}=$ $1,4 \mathrm{~m} / \mathrm{s}$

Figura 4.48 - Caso 4, Configuração 1 - Fração volumétrica do ar no plano de simetria (a), entrada (b) e saída (c) do misturador para $U_{m}=$ $2,0 \mathrm{~m} / \mathrm{s}$

Figura 4.49 - Caso 4, Configuração 2 - Fração volumétrica do ar no plano de simetria (a), entrada (b) e saída (c) do misturador para $U_{m}=$ $1,2 \mathrm{~m} / \mathrm{s}$ 
Figura 4.50 - Caso 4, Configuração 2 - Fração volumétrica do ar no plano de simetria (a), entrada (b) e saída (c) do misturador para $U_{m}=$ $1,4 \mathrm{~m} / \mathrm{s}$

Figura 4.51 - Caso 4, Configuração 2 - Fração volumétrica do ar no plano de simetria (a), entrada (b) e saída (c) do misturador para $U_{m}=$ $2,0 \mathrm{~m} / \mathrm{s}$

Figura 4.52 - Caso 4, Configuração 3 - Fração volumétrica do ar no plano de simetria (a), entrada (b) e saída (c) do misturador para $U_{m}=$ $1,2 \mathrm{~m} / \mathrm{s}$

Figura 4.53 - Caso 4, Configuração 3 - Fração volumétrica do ar no plano de simetria (a), entrada (b) e saída (c) do misturador para $U_{m}=$ $1,4 \mathrm{~m} / \mathrm{s}$

Figura 4.54 - Caso 4, Configuração 3 - Fração volumétrica do ar no plano de simetria (a), entrada (b) e saída (c) do misturador para $U_{m}=$ $2,0 \mathrm{~m} / \mathrm{s}$

Figura 4.55 - Caso 4, Configuração 4 - Fração volumétrica do ar no plano de simetria (a), entrada (b) e saída (c) do misturador para $U_{m}=$ $1,2 \mathrm{~m} / \mathrm{s}$

Figura 4.56 - Caso 4, Configuração 4 - Fração volumétrica do ar no plano de simetria (a), entrada (b) e saída (c) do misturador para $U_{m}=$ $1,4 \mathrm{~m} / \mathrm{s}$

Figura 4.57 - Caso 4, Configuração 4 - Fração volumétrica do ar no plano de simetria (a), entrada (b) e saída (c) do misturador para $U_{m}=$ $2,0 \mathrm{~m} / \mathrm{s}$

Figura 4.58 - Caso 4, Configuração 1 - Diâmetro representativo das bolhas no plano de simetria (a), entrada (b) e saída (c) do misturador para $U_{m}=1,2 \mathrm{~m} / \mathrm{s}$

Figura 4.59 - Caso 4, Configuração 1 - Diâmetro representativo das bolhas no plano de simetria (a), entrada (b) e saída (c) do misturador para $U_{m}=1,4 \mathrm{~m} / \mathrm{s}$

Figura 4.60 - Caso 4, Configuração 1 - Diâmetro representativo das bolhas no plano de simetria (a), entrada (b) e saída (c) do misturador para $U_{m}=2,0 \mathrm{~m} / \mathrm{s}$

Figura 4.61 - Caso 4, Configuração 2 - Diâmetro representativo das bolhas no plano de simetria (a), entrada (b) e saída (c) do misturador para $U_{m}=1,2 \mathrm{~m} / \mathrm{s}$

Figura 4.62 - Caso 4, Configuração 2 - Diâmetro representativo das bolhas no plano de simetria (a), entrada (b) e saída (c) do misturador para $U_{m}=1,4 \mathrm{~m} / \mathrm{s}$

Figura 4.63 - Caso 4, Configuração 2 - Diâmetro representativo das bolhas no plano de simetria (a), entrada (b) e saída (c) do misturador para $U_{m}=2,0 \mathrm{~m} / \mathrm{s}$ 
Figura 4.64 - Caso 4, Configuração 3 - Diâmetro representativo das bolhas no plano de simetria (a), entrada (b) e saída (c) do misturador para $U_{m}=1,2 \mathrm{~m} / \mathrm{s}$

Figura 4.65 - Caso 4, Configuração 3 - Diâmetro representativo das bolhas no plano de simetria (a), entrada (b) e saída (c) do misturador para $U_{m}=1,4 \mathrm{~m} / \mathrm{s}$

Figura 4.66 - Caso 4, Configuração 3 - Diâmetro representativo das bolhas no plano de simetria (a), entrada (b) e saída (c) do misturador para $U_{m}=2,0 \mathrm{~m} / \mathrm{s}$

Figura 4.67 - Caso 4, Configuração 4 - Diâmetro representativo das bolhas no plano de simetria (a), entrada (b) e saída (c) do misturador para $U_{m}=1,2 \mathrm{~m} / \mathrm{s}$

Figura 4.68 - Caso 4, Configuração 4 - Diâmetro representativo das bolhas no plano de simetria (a), entrada (b) e saída (c) do misturador para $U_{m}=1,4 \mathrm{~m} / \mathrm{s}$

Figura 4.69 - Caso 4, Configuração 4 - Diâmetro representativo das bolhas no plano de simetria (a), entrada (b) e saída (c) do misturador para $U_{m}=2,0 \mathrm{~m} / \mathrm{s}$

Figura 4.70 - Caso 4, Configuração 1 - Distribuição das bolhas dos 10 grupos no plano de simetria e saída do misturador para $U_{m}=$ $2,0 \mathrm{~m} / \mathrm{s}$

Figura 4.71 - Caso 4, Configuração 2 - Distribuição das bolhas dos 10 grupos no plano de simetria e saída do misturador para $U_{m}=$ $2,0 \mathrm{~m} / \mathrm{s}$

Figura 4.72 - Caso 4, Configuração 3 - Distribuição das bolhas dos 10 grupos no plano de simetria e saída do misturador para $U_{m}=$ $2,0 \mathrm{~m} / \mathrm{s}$

Figura 4.73 - Caso 4, Configuração 4 - Distribuição das bolhas dos 10 grupos no plano de simetria e saída do misturador para $U_{m}=$ $2,0 \mathrm{~m} / \mathrm{s}$

Figura 5.1 - Padrões de escoamento para tubulações horizontais (Fonseca Junior, 2009)

Figura 5.2 - Mapa de padrão de escoamento (Taitel e Dukler, 1976) 107

Figura 5.3 - Duas unidades de golfada

Figura 5.4 - Simulação de golfadas para a configuração 1 e $U_{m}=$ $1,4 \mathrm{~m} / \mathrm{s}$

Figura 5.5 - Configuração 1, comportamento da fração volumétrica de ar no interior do misturador com a passagem de uma golfada

Figura 5.6 - Diferencial de pressão durante a simulação de golfadas para a configuração 1 e $U_{m}=1,4 \mathrm{~m} / \mathrm{s}$

Figura 5.7 - Configuração 1, distribuição dos grupos de bolhas na saída ao final da passagem de uma bolha de ar (tempo =6,44s) 
Figura 5.8 - Simulação de golfadas para a configuração 3 e $U_{m}=$ $1,4 \mathrm{~m} / \mathrm{s}$

Figura 5.9 - Configuração 3, comportamento da fração volumétrica de ar no interior do misturador com a passagem de uma golfada

Figura 5.10 - Diferencial de pressão durante a simulação de golfadas para a configuração 3 e $U_{m}=1,4 \mathrm{~m} / \mathrm{s}$

Figura 5.11 - Configuração 3, distribuição dos grupos de bolhas na saída ao final da passagem de uma bolha de ar (tempo $=6,08 \mathrm{~s}$ )

Figura 5.12 - Caso extremo, 98\% de fração de ar na entrada, para a configuração 1 e $U_{m}=1,4 \mathrm{~m} / \mathrm{s}$

Figura 5.13 - Caso extremo, 98\% de fração de ar na entrada, para a configuração 3 e $U_{m}=1,4 \mathrm{~m} / \mathrm{s}$

Figura 5.14 - Configuração proposta (a), configuração 1 (b) e configuração 3 (c)

Figura 5.15 - Simulação de golfadas para a configuração proposta

Figura 5.16 - Diferencial de pressão durante a simulação de golfadas para a configuração proposta

Figura 5.17 - Configuração proposta, comportamento da fração volumétrica de ar no interior do misturador com a passagem de uma golfada

Figura 5.18 - Configuração proposta, distribuição dos grupos de bolhas na saída no pico de fração de ar na saída (tempo = 6,67s)

Figura 5.19 - Caso extremo, configuração proposta, $98 \%$ de fração de ar na entrada e $U_{m}=1,4 \mathrm{~m} / \mathrm{s}$

Figura 5.20 - Fração de ar e diâmetro representativo das bolhas de ar, configuração proposta, injeção de somente ar (fração de $98 \%$ ), tempo $=0,0 \mathrm{~s}$

Figura 5.21 - Fração de ar e Diâmetro representativo das bolhas de ar na configuração proposta, injeção de somente ar (fração de 98 $\%)$, tempo = 1,00s; 2,00 s e $2,93 \mathrm{~s}$

Figura 5.22 - Fração de ar e diâmetro representativo das bolhas de ar na configuração proposta, injeção de somente ar (fração de 98 $\%)$, tempo $=3,33 \mathrm{~s}$

Figura 5.23 - Fração de ar e diâmetro representativo das bolhas de ar na configuração proposta, injeção de somente ar (fração de 98 $\%)$, tempo $=3,93 \mathrm{~s}$ e $=5,59 \mathrm{~s}$ 


\section{Lista de símbolos}

$A_{\alpha \beta} \quad$ área superficial de uma partícula esférica da fase $\beta$, por unidade de volume

$a_{1} \quad$ constante empírica do modelo de turbulência

$B_{B}$ taxa de nascimento de bolhas devido à quebra de bolhas grandes

$D_{B} \quad$ taxa de morte devido à quebra em bolhas menores

$B_{C} \quad$ taxa de nascimento devido à coalescência de bolhas pequenas

$D_{C} \quad$ taxa de morte devido à coalescência com outras bolhas

$C_{D} \quad$ Coeficiente de arraste adimensional

$C D_{k \omega} \quad$ parte positiva do termo de difusão cruzada

$C_{L} \quad$ Coeficiente de sustentação

$d_{\beta} \quad$ diâmetro médio das partículas esféricas da fase $\beta$

$d_{b} \quad$ diâmetro da bolha

$D_{p} \quad$ diâmetro da partícula

Eo número de Eötvös

$F_{B} \quad$ coeficiente de calibração do modelo de quebra de bolhas

$F_{C B} \quad$ coeficiente de calibração da contribuição da flutuação do modelo de coalescência de bolhas

$F_{C T} \quad$ coeficiente de calibração da contribuição turbulenta do modelo de coalescência de bolhas

$\vec{F}_{L} \quad$ força de sustentação de uma partícula

$F_{1} \quad$ função de mistura

$F_{2} \quad$ função de mistura para a viscosidade turbulenta

$g \quad$ aceleração da gravidade

$h_{0} \quad$ espessura inicial do filme de líquido

$h_{f} \quad$ espessura crítica onde a ruptura do filme de líquido ocorre

$k \quad$ energia cinética turbulenta

$m \quad$ massa

$M_{\alpha} \quad$ força total imposta à fase $\alpha$ devido à interação com outras fases. 
$M_{\alpha \beta}^{D} \quad$ força de arraste

$M_{\alpha \beta}^{L} \quad$ força de sustentação

$M_{\alpha \beta}^{L U B} \quad$ força de lubrificação da parede

$M_{\alpha \beta}^{V M} \quad$ força de massa virtual

$M_{\alpha \beta}^{T D} \quad$ força de dispersão turbulenta

Mo número de Morton

$p_{\alpha} \quad$ pressão da fase $\alpha$

$r_{\alpha} \quad$ fração volumétrica da fase $\alpha$

$r_{l} \quad$ fração volumétrica da fase líquido (contínua)

$r_{g} \quad$ fração volumétrica da fase gás (dispersa)

$r_{d m} \quad$ valor máximo de empacotamento

Re Número de Reynolds

$R_{m} \quad$ Número de Reynolds modificado

Número de Reynolds da partícula

$R_{s} \quad$ Número de Reynolds devido à taxa de deformação do escoamento

$r_{i j} \quad$ raio equivalente

$S_{M \alpha} \quad$ representa fontes de quantidade de movimento devido à forças externas

$S_{M S \alpha} \quad$ possíveis fontes de massa da fase $\alpha$

$S_{i j} \quad$ área da seção transversal das partículas em colisão

$S \quad$ módulo do tensor deformação do escoamento

$\overrightarrow{U_{\alpha}} \quad$ vetor velocidade da fase $\alpha$

$\overrightarrow{U_{l}} \quad$ vetor velocidade da fase líquida

$\overrightarrow{U_{g}} \quad$ vetor velocidade da fase gás

$\overrightarrow{U_{p}} \quad$ vetor velocidade de uma partícula

$\overrightarrow{U_{f}} \quad$ vetor velocidade do fluido

$t_{i j} \quad$ tempo requerido para a coalescência de duas bolhas

$u_{t i} \quad$ velocidade turbulenta

$\forall \quad$ volume

$y \quad$ distância à parede 


\section{Símbolos gregos}

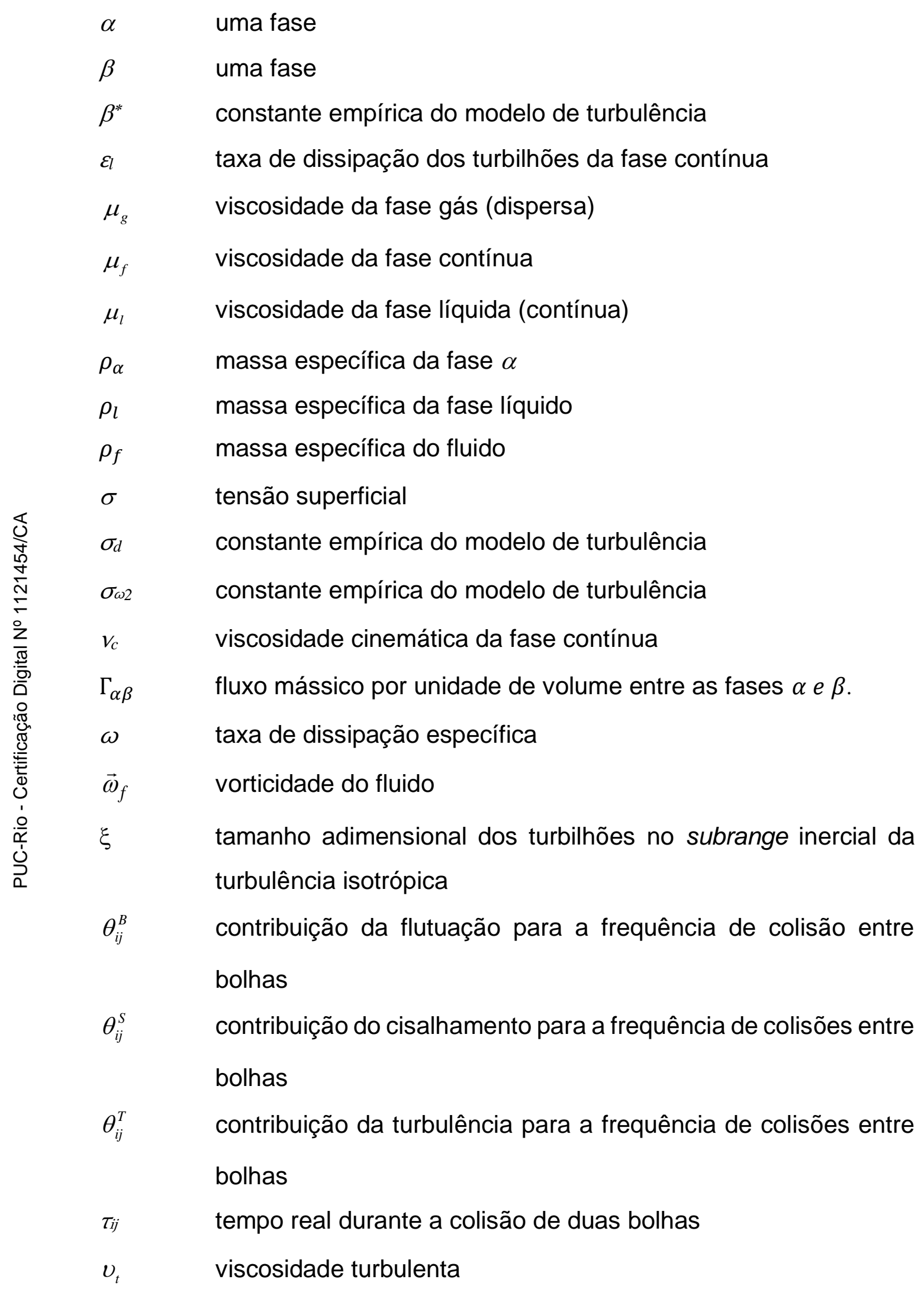


"O aumento do conhecimento é como uma esfera dilatando-se no espaço: quanto maior a nossa compreensão, maior o nosso contato com o desconhecido." Blaise Pascal 